\title{
A Novel Time-Frequency Technique for Mode Retrieval Based on Linear Chirp
}

\section{Approximation}

\author{
Nils Laurent, Sylvain Meignen
}

\begin{abstract}
In this paper, we introduce a novel time-frequency technique for the retrieval of the modes of multicomponent signals based on linear chirp approximation. The key idea to this new technique is to design the retrieval procedure by using only information extracted in the vicinity of the ridges made by the components in the time-frequency plane. Compared with state-of-the-art methods based on timefrequency representations, the proposed approach will prove to improve the reconstruction results when applied to a monocomponent signal and to circumvent the mode-mixing issue when the modes of a multicomponent signal are close in the time-frequency plane.
\end{abstract}

\section{Index Terms}

Time-frequency, AM/FM multicomponent signal, mode retrieval, linear chirp approximation.

\section{INTRODUCTION}

Multicomponent signals (MCSs) have received a lot of interest in the last decades since they enable to accurately represent non-stationary signals encountered in many different fields such as for instance pathology diagnosis [1], [2], structural damage [3], [4] or physiological signals [5]. The short-time Fourier transform (STFT) is a time-frequency representation (TFR) very commonly used to analyze this type of signals because the modes making up MCSs are associated with specific regions in the time-frequency (TF) plane, centered around so-called ridges [6].

The authors are with the Jean Kuntzmann Laboratory, University Grenoble Alpes and CNRS 5225, Grenoble 38401, France (emails: nils.laurent@univ-grenoble-alpes.fr sylvain.meignen@univ-grenoble-alpes.fr). This work was supported in part by the University Grenoble Alpes under IRS Grant "AMUSETE" and the ANR ASCETE project with grant number ANR-19-CE480001-01. 
In this context, mode retrieval is basically carried out by determining the specific $\mathrm{TF}$ region associated with each mode. For that purpose, different non parametric techniques were developed either inspired by hard-thresholding approaches [7], as for instance in [8] and more recently in [9], or based on synchrosqueezing transform [10], [11]. As we will recall, these techniques basically consist of cleverly summing the coefficients of the STFT belonging to the TF region associated with each mode, but when some modes exhibit interference in the TF plane, these regions overlap, leading to mode-mixing. Remarking that these interference have a smaller impact at TF locations corresponding to high STFT modulus, we propose to design a new mode retrieval technique from the estimation of the STFT using only information extracted in the vicinity of the ridges associated with the modes. We will show, on different types of simulated signals, that the proposed approach considerably reduces mode-mixing compared with the above mentioned state-of-the-art TF based methods. At this point, it is important to note that there exist a series of other techniques that intend to retrieve the modes without considering TFRs, like the chirplet transform [12] or ridgelet transform [13] which fundamentally differ from the above mentioned techniques in that they are parametric and non local, therefore we will stick to TF-based methods in terms of comparisons.

After having recalled basic notations and definitions in Section [II, we introduce our new approach for mode retrieval in Section III, then recall briefly alternative techniques based either on hard-thresholding and variants [7] [9], or second order synchrosqueezing transform [11] in Section IV] These are finally compared to our new approach in Section $\mathrm{V}$.

\section{Definitions And Notations}

The short-time Fourier transform (STFT) is defined for $f \in L^{1}(\mathbb{R}), g \in L^{\infty}(\mathbb{R})$ as $[14]$ :

$$
V_{f}^{g}(t, \eta)=\int_{\mathbb{R}} f(\tau) g(\tau-t) e^{-2 i \pi \eta(\tau-t)} d \tau .
$$

Assuming $g$ is real such that $g(0) \neq 0$, then the signal $f$ can be retrieved through:

$$
f(t)=\frac{1}{g(0)} \int_{\mathbb{R}} V_{f}^{g}(t, \eta) d \eta .
$$

Assuming $f$ and $g \in L^{2}(\mathbb{R})$ such that $\|g\|_{2}=1$, one has the alternative reconstruction formula:

$$
f(t)=\iint_{\mathbb{R}^{2}} V_{f}^{g}(u, \eta) g(t-u) e^{i 2 \pi \eta(t-u)} d u d \eta .
$$

In this paper we will make extensive use of MCSs corresponding to the superimposition of $P$ modes defined as:

$$
f(t)=\sum_{p=1}^{P} f_{p}(t)
$$


with $f_{p}(t)=A_{p}(t) e^{2 i \pi \phi_{p}(t)}$, in which the instantaneous amplitude (IA) $A_{p}(t)>0$ varies slowly and the instantaneous frequency (IF) $\phi_{p}^{\prime}(t)>0$ for all $p$, and such that $\phi_{p+1}^{\prime}(t)-\phi_{p}^{\prime}(t)>0$. We further assume the modes are separated in frequency with resolution $\Delta$ :

$$
\forall 1 \leq p \leq P-1, \phi_{p+1}^{\prime}(t)-\phi_{p}^{\prime}(t)>2 \Delta .
$$

In the TF plane, the different modes are associated with so-called ridges, which we denote $\left(\varphi_{p}\right)_{p=1, \cdots, P}$, that are estimates of the IFs $\left(\phi_{p}^{\prime}\right)_{p=1, \cdots, P}$ of the modes. Since, ridge extraction is not the scope of the present paper, we will not focus on this issue and refer the reader to the vast literature on the subject [8], [15]-[17], and adopt, in the present paper, the approach of [8] .

\section{Mode Retrieval Using STFT Estimation BAsed on Linear Chirp Approximation}

Our goal is now to introduce a novel technique to retrieve the modes of a MCS using a local approximation of its STFT assuming a mode locally behaves like a linear chirp. This is done in Section III-A. Section III-B then focuses on the practical implementation of the proposed technique.

\section{A. Principles of the Novel Mode Retrieval Technique}

Let us assume that $g$ in (1) is the Gaussian window $g(t)=e^{-\pi \frac{t^{2}}{\sigma^{2}}}$, the parameter $\sigma$ being chosen so as to minimize the Rényi entropy associated with the STFT modulus, as is done in [8], [18]. Then, as shown for instance in [19], the STFT of the linear chirp $f(t)=A e^{2 i \pi\left(a t+b \frac{t^{2}}{2}\right)}$ can be expressed as:

$$
\begin{aligned}
V_{f}^{g}(t, \eta) & =\quad A r^{-\frac{1}{2}} e^{-i \frac{\theta}{2}} e^{-\frac{\pi \sigma^{2}\left(1+i b \sigma^{2}\right)}{1+\left(b \sigma^{2}\right)^{2}}(\eta-a-b t)^{2}} \\
& =\quad V_{f}^{g}(t, a+b t) e^{\frac{-\pi \sigma^{2}\left(1+i b \sigma^{2}\right)}{1+\left(b \sigma^{2}\right)^{2}}(\eta-a-b t)^{2}} \\
& =V_{f}^{g}\left(t, \phi^{\prime}(t)\right) e^{\frac{-\pi \sigma^{2}\left(1+i \phi^{\prime \prime}(t) \sigma^{2}\right)}{1+\left(\phi^{\prime \prime}(t) \sigma^{2}\right)^{2}}\left(\eta-\phi^{\prime}(t)\right)^{2}},
\end{aligned}
$$

with $r=\sqrt{\frac{1}{\sigma^{4}}+\phi^{\prime \prime}(t)^{2}}$ and $\theta=\tan ^{-1}\left(-\phi^{\prime \prime}(t) \sigma^{2}\right)$. If the monocomponent signal $f$ departs from a linear chirp, the above equalities become approximations, which are furthermore only valid for $\eta$ in the vicinity of $\phi^{\prime}(t)$. Applying this approximation to $f_{p}$, we obtain for $\eta$ in the vicinity of $\phi_{p}^{\prime}(t)$ :

$$
\begin{aligned}
V_{f_{p}}^{g}(t, \eta) & \approx V_{f_{p}}^{g}\left(t, \phi_{p}^{\prime}(t)\right) e^{\frac{-\pi \sigma^{2}\left(1+i \phi_{p}^{\prime \prime}(t) \sigma^{2}\right)}{1+\left(\phi_{p}^{\prime \prime}(t) \sigma^{2}\right)^{2}}\left(\eta-\phi_{p}^{\prime}(t)\right)^{2}} \\
& \approx V_{f}^{g}\left(t, \phi_{p}^{\prime}(t)\right) e^{\frac{-\pi \sigma^{2}\left(1+i \phi_{p}^{\prime \prime}(t) \sigma^{2}\right)}{1+\left(\phi_{p}^{\prime \prime}(t) \sigma^{2}\right)^{2}}\left(\eta-\phi_{p}^{\prime}(t)\right)^{2}} .
\end{aligned}
$$

In the last equation we have replaced $V_{f_{p}}^{g}\left(t, \phi_{p}^{\prime}(t)\right)$ by $V_{f}^{g}\left(t, \phi_{p}^{\prime}(t)\right)$, remarking that at TF location $\left(t, \phi_{p}^{\prime}(t)\right)$, the STFT of the mode $f_{q}, q \neq p$ is close to 0 . 
In practice, the IF $\phi_{p}^{\prime}(t)$ and the chirp rate $(\mathrm{CR}) \phi_{p}^{\prime \prime}(t)$ are to be estimated. For that purpose, we use the estimates involved in the definition of the second order synchrosqueezing transform (SST2) [19]. For CR estimation, the following complex modulation operator was introduced:

$$
\tilde{q}_{f}(t, \eta)=\frac{1}{2 i \pi} \frac{V_{f}^{g^{\prime \prime}}(t, \eta) V_{f}^{g}(t, \eta)-\left(V_{f}^{g^{\prime}}(t, \eta)\right)^{2}}{V_{f}^{t g}(t, \eta) V_{f}^{g^{\prime}}(t, \eta)-V_{f}^{t g^{\prime}}(t, \eta) V_{f}^{g}(t, \eta)},
$$

which is such that $\widehat{q}_{f}(t, \eta)=\Re\left\{\tilde{q}_{f}(t, \eta)\right\}=\phi^{\prime \prime}(t)$ when $f$ is a linear chirp. In the context of a MCS, $\widehat{q}_{f}(t, \eta) \approx \phi_{p}^{\prime \prime}(t)$ where $\eta$ is close to $\phi_{p}^{\prime}(t)$, therefore we consider $\widehat{q}_{f}\left(t, \varphi_{p}(t)\right)$ to estimate $\phi_{p}^{\prime \prime}(t)$, bearing in mind that $\left(t, \varphi_{p}(t)\right)$ corresponds to the ridge associated with $f_{p}$ and thus $\varphi_{p}$ is an estimate of its IF. Then, introducing $\tilde{\omega}_{f}(t, \eta)=\eta-\frac{1}{2 i \pi} \frac{V_{f}^{g^{\prime}}(t, \eta)}{V_{f}^{g}(t, \eta)}$ and $\tau(t, \eta)=-\frac{V_{f}^{t g}(t, \eta)}{V_{f}^{g}(t, \eta)}$,

$$
\widehat{\omega}_{f}^{[2]}(t, \eta)=\Re\left\{\tilde{\omega}_{f}(t, \eta)-\tilde{q}_{f}(t, \eta) \tau(t, \eta)\right\},
$$

was defined and equals $\phi^{\prime}(t)$ when $f$ is a linear chirp [11]. In the context of a MCS, $\widehat{\omega}_{f}^{[2]}(t, \eta) \approx \phi_{p}^{\prime}(t)$ when $\eta$ is close to $\phi_{p}^{\prime}(t)$, therefore we consider $\widehat{\omega}_{f}^{[2]}\left(t, \varphi_{p}(t)\right)$ to estimate $\phi_{p}^{\prime}(t)$. Then, from (7) the STFT of $f_{p}$ can be approximated by :

$$
\begin{array}{r}
V_{f_{p}}^{g}(t, \eta) \approx \\
V_{f}^{g}\left(t, \widehat{\omega}_{f}^{[2]}\left(t, \varphi_{p}(t)\right)\right) e^{-\frac{\pi \sigma^{2}\left(1+i \widehat{q}_{f}\left(t, \varphi_{p}(t)\right) \sigma^{2}\right)}{1+\left(\widehat{q}_{f}\left(t, \varphi_{p}(t)\right) \sigma^{2}\right)^{2}}\left(\eta-\widehat{\omega}_{f}^{[2]}\left(t, \varphi_{p}(t)\right)\right)^{2}} .
\end{array}
$$

Finally, the reconstruction of mode $f_{p}$ is carried out either through (2) or 3 replacing $V_{f}^{g}$ by the approximation of $V_{f_{p}}^{g}$ given by (10). In noisy cases, the signal $f$ is replaced by $\tilde{f}:=f+\varepsilon$, where $\varepsilon$ is some noise, and the approximation of $V_{f_{p}}^{g}$ is obtained by replacing $f$ by $\tilde{f}$ in $(10)$, the reconstruction procedures being then the same as in the noise-free case.

It is important to remark here that the approximation of $V_{f_{p}}^{g}$ uses only STFTs of $f$ evaluated in the vicinity of the ridge associated with $f_{p}$, which will prove to be the very reason why the just described approach for mode retrieval is well adapted to handle the mode-mixing issue. However, up to now, we have only presented the approach for continuous time signals, and it needs to be adapted to discrete time signals to be of practical use, as explained hereafter.

\section{B. Practical Implementation}

To implement the just described mode retrieval technique, one considers that the signal $f$ is a discrete sequence of length $L$ such that $f[n]=f\left(\frac{n}{L}\right)$, for $n=0, \cdots, L-1$, and that $(g[n])_{n \in \mathbb{Z}}$ are the samples at $\frac{n}{L}$ of the Gaussian window, which is further truncated to be supported on $\{-M, \cdots, M\}$ such that 
$2 M+1 \leq N$, where $N$ is the number of frequency bins. In that context, the discrete STFT of $f$ is defined by [20]

$$
\begin{aligned}
\mathbf{V}_{f}^{g}[m, k] & =\sum_{n \in \mathbb{Z}} f[n] g[n-m] e^{-2 i \pi \frac{k(n-m)}{N}} \\
& =\sum_{n=-M}^{n=M} f[m+n] g[n] e^{-2 i \pi \frac{k n}{N}}
\end{aligned}
$$

with $k \in\{0, \cdots, N-1\}$, and $m \in\{0, \cdots, L-1\}$, which can be computed for each $m$ through a discrete Fourier transform of length $N$. Note that the index $k$ corresponds to a frequency in $I_{L}=$ $\left\{0, \frac{L}{N} \cdots,(N-1) \frac{L}{N}\right\}$, and the index $n$ to time $\frac{n}{L}$. In that context, the discrete mode $\left(f_{p}[m]\right)_{m}$ is associated with a ridge $\left(\varphi_{p}[m]\right)_{m}$ corresponding to frequency indices in $\{0, \cdots, N-1\}$. Discrete versions of $\widehat{\omega}_{f}^{[2]}$ and $\widehat{q}_{f}$ can be computed and are denoted by $\widehat{\omega}_{f}^{[2]}[m, k]$ and $\widehat{q}_{f}[m, k]$ in the sequel, enabling us to define $\psi_{p}^{\prime}[m]:=\widehat{\omega}_{f}^{[2]}\left[m, \varphi_{p}[m]\right]$ and $\psi_{p}^{\prime \prime}[m]:=\widehat{q}_{f}\left[m, \varphi_{p}[m]\right]$ approximating $\phi_{p}^{\prime}\left(\frac{m}{L}\right)$ and $\phi_{p}^{\prime \prime}\left(\frac{m}{L}\right)$ respectively. Remarking, using rectangular integration, that $V_{f}^{g}\left(\frac{m}{L}, k \frac{L}{N}\right) \approx \frac{\mathbf{V}_{f}^{g}[m, k]}{L}$, and using (7), we may write:

$$
\begin{aligned}
\mathbf{V}_{f_{p}}^{g}[m, k] & \approx L V_{f_{p}}^{g}\left(\frac{m}{L}, k \frac{L}{N}\right) \\
& \approx L V_{f}^{g}\left(\frac{m}{L}, \phi_{p}^{\prime}\left(\frac{m}{L}\right)\right) e^{-\frac{\pi \sigma^{2}\left(1+i \phi_{p}^{\prime \prime}\left(\frac{m}{L}\right) \sigma^{2}\right)}{1+\left(\phi_{p}^{\prime \prime}\left(\frac{m}{L}\right) \sigma^{2}\right)^{2}}\left(\frac{k L}{N}-\phi_{p}^{\prime}\left(\frac{m}{L}\right)\right)^{2}} \\
& \approx L V_{f}^{g}\left(\frac{m}{L}, \psi_{p}^{\prime}[m]\right) e^{\left.-\frac{\pi \sigma^{2}\left(1+i \psi_{p}^{\prime \prime}[m] \sigma^{2}\right)}{1+\left(\psi_{p}^{\prime \prime}[m] \sigma^{2}\right)^{2}}\left(\frac{k L}{N}-\psi_{p}^{\prime}[m]\right)\right)^{2}}
\end{aligned}
$$

for which an approximation of $V_{f}^{g}\left(\frac{m}{L}, \psi_{p}^{\prime}[m]\right)$ from values of the discrete STFT is needed. For that purpose, let us consider $k_{0}:=\left\lfloor\psi_{p}^{\prime}[m] \frac{N}{L}\right\rceil$, where $\lfloor x\rceil$ denotes the closest integer to $x$, we may write using (10):

$$
\begin{aligned}
V_{f}^{g}\left(\frac{m}{L}, \psi_{p}^{\prime}[m]\right) & \approx V_{f_{p}}^{g}\left(\frac{m}{L}, \psi_{p}^{\prime}[m]\right) \\
& \approx V_{f}^{g}\left(\frac{m}{L}, k_{0} \frac{L}{N}\right) e^{\frac{\pi \sigma^{2}\left(1+i \psi_{p}^{\prime \prime}[m] \sigma^{2}\right)}{1+\left(\psi_{p}^{\prime \prime}[m] \sigma^{2}\right)^{2}}\left(k_{0} \frac{L}{N}-\psi_{p}^{\prime}[m]\right)^{2}} \\
& \approx \frac{1}{L} \mathbf{V}_{f}^{g}\left[m, k_{0}\right] e^{\frac{\pi \sigma^{2}\left(1+i \psi_{p}^{\prime \prime}[m] \sigma^{2}\right)}{1+\left(\psi_{p}^{\prime \prime}[m] \sigma^{2}\right)^{2}}\left(k_{0} \frac{L}{N}-\psi_{p}^{\prime}[m]\right)^{2}} .
\end{aligned}
$$

From (12) and (13) we get that:

$$
\begin{aligned}
& \mathbf{V}_{f_{p}}^{g}[m, k] \\
\approx & \mathbf{V}_{f}^{g}\left[m, k_{0}\right] e^{\frac{\pi \sigma^{2}\left(1+i \psi_{p}^{\prime \prime}[m] \sigma^{2}\right)}{1+\left(\psi_{p}^{\prime \prime}[m] \sigma^{2}\right)^{2}}\left[\left(\frac{k_{0} L}{N}-\psi_{p}^{\prime}[m]\right)^{2}-\left(\frac{k L}{N}-\psi_{p}^{\prime}[m]\right)^{2}\right]} \\
\approx & \mathbf{V}_{f}^{g}\left[m, k_{0}\right] e^{\frac{\pi \sigma^{2}\left(1+i \psi_{p}^{\prime \prime}[m] \sigma^{2}\right)}{1+\left(\psi_{p}^{\prime \prime}[m] \sigma^{2}\right)^{2}}\left[\frac{L\left(k_{0}-k\right)}{N}\left(\frac{L\left(k_{0}+k\right)}{N}-2 \psi_{p}^{\prime}[m]\right)\right]} .
\end{aligned}
$$

If we denote $\tilde{\mathbf{V}}_{f_{p}}^{g}$ the estimation of $\mathbf{V}_{f_{p}}^{g}$ given by (14), the retrieval of $f_{p}$ can be carried out either through:

$$
f_{p}[m] \approx \frac{1}{g(0) N} \sum_{k=0}^{N-1} \tilde{\mathbf{V}}_{f_{p}}^{g}[m, k]
$$


or through, assuming $f$ is L-periodic:

$$
f_{p}[m] \approx \frac{\sum_{q=m-M}^{m+M} \sum_{k=0}^{N-1} \tilde{\mathbf{V}}_{f_{p}}^{g}[q \bmod L, k] g[m-q] \frac{e^{i 2 \pi \frac{k(m-q)}{N}}}{N}}{\sum_{q=m-M} g[m-q]^{2}} .
$$

In a noisy context, all the above expressions can be used replacing $f$ by its noisy version $\tilde{f}$. These new techniques to retrieve the different modes are called Linear Chirp based Retrieval (LCR).

\section{Alternative Techniques for mode Rerieval: HT, SSR-HT [9], AND SYNhrosqueEZING TRANSFORMS [11]}

The Hard Thresholding (HT) technique when used for the retrieval of the mode $f_{p}$ of a MCS [8] consists of considering in the reconstruction process only the coefficients of $\mathbf{V}_{\tilde{f}}^{g}$ in the vicinity of $\tilde{\varphi}_{p}$, the ridge associated with $\tilde{f}_{p}$ the noisy version of $f_{p}$. For that purpose, one defines for each time indexed by $m$, an interval $J_{p}[m]=\left[\eta_{p}^{-}[m], \eta_{p}^{+}[m]\right]$ such that:

$$
\begin{aligned}
& \eta_{p}^{-}[m]:=\underset{k}{\operatorname{argmax}}\left\{\frac{k L}{N}<\tilde{\varphi}_{p}[m],\left|\mathbf{V}_{\tilde{f}}^{g}[m, k]\right|<3 \sigma\|g\|_{2}\right\} \\
& \eta_{p}^{+}[m]:=\underset{k}{\operatorname{argmin}}\left\{\frac{k L}{N}>\tilde{\varphi}_{p}[m],\left|\mathbf{V}_{\tilde{f}}^{g}[m, k]\right|<3 \sigma\|g\|_{2}\right\},
\end{aligned}
$$

which depends on an estimate of $\gamma:=\sigma\|g\|_{2}$, for which a median absolute deviation approach [21] is used:

$$
\hat{\gamma}:=\frac{\operatorname{median}\left|\Re\left\{\mathbf{V}_{\tilde{f}}^{g}[m, k]\right\}_{m, k}\right|}{0.6745} .
$$

The reconstruction of the modes is carried out using only, in (15) or [16, the frequency indices in $J_{p}[m]$ for each $m$.

For the sake of comparison, we also briefly recall the principles of a denoising technique called Shifted-Symmetrized-Regularized Hard-Thresholding (SSR-HT), and introduced in [9], which consists of an improvement of HT technique based on a linear chirp approximation for the mode, that uses symmetry properties of the STFT with respect to the location of the local maxima of its modulus and also the hypothesis that the STFT is regular.

We would like to compare also LCR to the second-order synchrosqueezing transform (SST2) with accuracy $\alpha$ which was defined in [11], in the continuous time context, as:

$$
T V_{f}^{g}(t, \omega)=\int_{\left|V_{f}^{g}(t, \eta)\right| \geq \alpha} V_{f}^{g}(t, \eta) \delta\left(\omega-\widehat{\omega}_{f}^{[2]}(t, \eta)\right) d \eta,
$$


in which $\widehat{\omega}_{f}^{[2]}(t, \eta)=\Re\left\{\tilde{\omega}_{f}^{[2]}(t, \eta)\right\}$, and $\delta$ the Dirac distribution. The reconstruction of mode $p$ being then achieved through:

$$
f_{p}(t) \approx \frac{1}{g(0)} \int_{\left|\omega-\varphi_{p}(t)\right| \leq d} T V_{f}^{g}(t, \omega) d \omega
$$

where $d$ is a parameter to account for errors in evaluating the IF of $f_{p}$ with the ridge $\varphi_{p}$. Note that in SST2, the ridges are computed on the modulus of the synchrosqueezed transform $T V_{f}^{g}$ and not on the modulus of STFT. Of course we will compare LCR with a discrete time version of SST2, which we do not detail here due to format constraints (see [11] for details).

\section{RESUlTS}

The objectives of the simulations we carry out are two-fold. The first one is to compare LCR with other types of mode retrieval techniques based on TFR recalled above, namely HT, SSR-HT and SST2, on monocomponent signals. The second one is to investigate the ability offered by the different methods to accurately separate two close modes in a MCS (the code to reproduce the figures is available at https://github.com/Nils-Laurent/LCR).

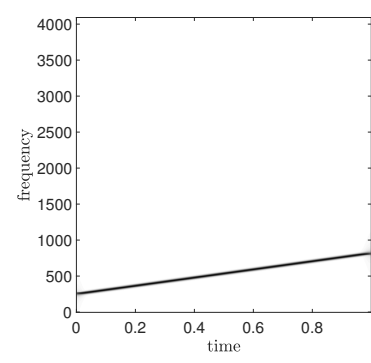

(a)

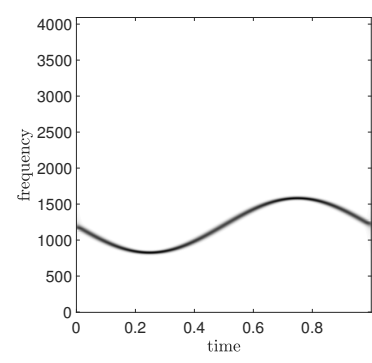

(b)

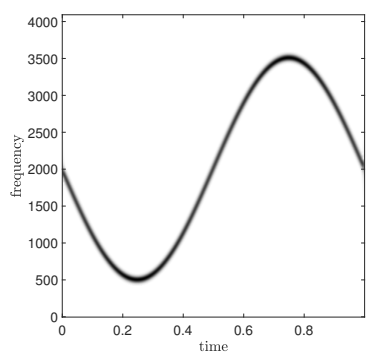

(c)

Fig. 1: (a): STFT of $f(t)=e^{2 i \pi\left(250 t+568 \frac{t^{2}}{2}\right)} ;$ (b): STFT of $f(t)=e^{2 i \pi(1200 t+60 \cos (3 \pi t))}$; (c): STFT of $f(t)=e^{2 i \pi(2000 t+238 \cos (2 \pi t))}$.

\section{A. Comparison of Mode Retrieval Techniques on a Monocomponent Signal}

Before we start the comparison of the different mode retrieval techniques, we numerically checked that the quality of IF estimate $\widehat{\omega}_{\tilde{f}}^{[2]}$ does not depend on the chirp rate for a linear chirp, whatever the noise level, and that this IF estimate is always more relevant than $\widehat{\omega}_{\tilde{f}}:=\Re\left\{\tilde{\omega}_{\tilde{f}}\right\}$ used in the original synchrosqueezing transform [10]. Then, we investigate the behavior of the different mode retrieval techniques for the modes depicted in Fig. 1. For that purpose, we compute the output SNR, i.e. 


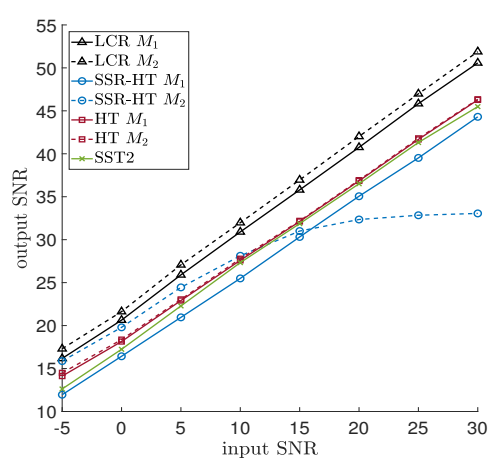

(a)

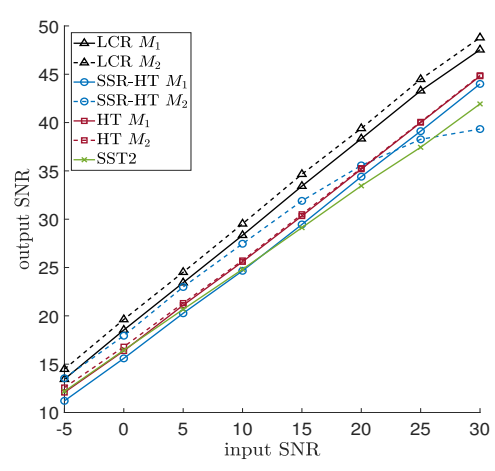

(b)

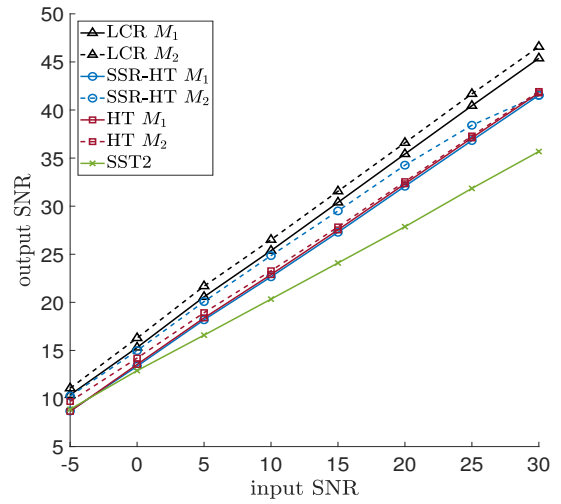

(c)

Fig. 2: (a): Signal reconstruction results for the signal of Fig. 1 (a) using HT, SSR-HT, SST2 (with $d=5$ ) and LCR. the mode is reconstructed using either (15) or 16 ; (b): Same as (a) but for the signal depicted in Fig. 1 (b); (c): Same as (a) but for the signal depicted in Fig. 11(c).

$S N R\left(f, f_{\text {rec }}\right)=20 \log _{10}\left(\|f\| /\left\|f_{\text {rec }}-f\right\|\right)$, with respect to input SNR, i.e. $S N R(f, \tilde{f})$ and where $f_{\text {rec }}$ corresponds to signal reconstruction with either (15) or 16). In Fig. 2, the results corresponding to the first (resp. second) reconstruction technique are denoted by $M_{1}$ (resp. $M_{2}$ ). We notice that LCR behaves better than HT, SSR-HT and SST2 in each case, whatever the noise level. We also note that LCR is only slightly sensitive to the technique used for mode reconstruction $\left(M_{2}\right.$ seems a little bit better than $M_{1}$, but the latter is much faster than the former). On the contrary, SSR-HT behaves worse when the modulation decreases, since the quality of reconstruction is hampered by frequency resolution when $M_{2}$ is used. Finally, comparing the results of Fig. 2 (b) and (c), we notice that LCR is performant for a wide range of frequency modulation for the mode.

\section{B. Mode Mixing Issue}

To tackle the mode mixing issue, we consider the two mode signal whose STFT is depicted in Fig. 3 (a), for which mode separation is challenging. In particular, we notice that at a low noise level and when using HT or SSR-HT, the threshold used for the determination of intervals $J_{p}$ is lower, at many time instants, than the minimum value of the modulus of STFT between the two ridges associated with the modes. This results in mode mixing with these two techniques. In Fig. 3 (b), we display the results corresponding to the retrieval of the modes $f_{1}$ and $f_{2}$. With HT and SSR-HT techniques, when the noise level is low, we get that for many time index $m, J_{1}[m]=J_{2}[m]$, hence the poor results. For SST2, with $d=5$, the quality of reconstruction is the same for the two studied modes, but the coefficients between 




(a)



(b)

Fig. 3: (a): STFT of signal made of two close chirps; (b): Reconstruction results using (15) for each of the two modes depicted in (a)

the modes are either used in the reconstruction of $f_{1}$ or $f_{2}$ while these coefficients contain information on both $f_{1}$ and $f_{2}$. This is the reason why when the noise level decreases the quality of reconstruction reaches a plateau with SST2. Note that, to change the value for $d$ would lead to the same conclusion. On the contrary, the separation of the two modes with LCR remains satisfactory whatever the noise level. It is worth mentioning here that the reconstruction formula used with HT, SSR-HT, and LCR is (15) because it is the closest to the one used in SST2. To use (16) instead would not change the conclusions.

\section{CONCLUSION}

In this paper, we have introduced a new technique for the reconstruction of the modes of multicomponent signals based on linear chirp approximation. We have shown that the proposed technique behaves better than commonly used hard-thresholding techniques or second order synchrosqueezing transform on a single mode signal and that it is much more performant than these techniques for separating two close modes. Future work should involve the extension of such a technique to the analysis of crossing modes or modes with highly oscillating phase, using higher order instantaneous frequency estimators. In another direction, we will investigate how to improve even more the robustness of the CR estimate used in the method when, in the signal, both the frequency modulation and the noise level are high.

\section{REFERENCES}

[1] U. R. Acharya, K. P. Joseph, N. Kannathal, L. C. Min, and J. S. Suri, "Heart rate variability," in Advances in cardiac signal processing. Springer, 2007, pp. 121-165.

[2] M. Malik and A. J. Camm, Dynamic electrocardiography. John Wiley \& Sons, 2008.

[3] M. Costa, A. A. Priplata, L. A. Lipsitz, Z. Wu, N. E. Huang, A. L. Goldberger, and C.-K. Peng, "Noise and poise: Enhancement of postural complexity in the elderly with a stochastic-resonance-based therapy," Europhysics Letters (EPL), vol. 77, no. 6, p. 68008, Mar 2007. 
[4] D. A. Cummings, R. A. Irizarry, N. E. Huang, T. P. Endy, A. Nisalak, K. Ungchusak, and D. S. Burke, “Travelling waves in the occurrence of dengue haemorrhagic fever in Thailand," Nature, vol. 427, no. 6972, pp. 344 -347, Jan 2004.

[5] C. L. Herry, M. Frasch, A. J. Seely, and H.-T. Wu, "Heart beat classification from single-lead ecg using the synchrosqueezing transform," Physiological Measurement, vol. 38, no. 2, pp. 171-187, 2017.

[6] P. Flandrin, Time-frequency/time-scale analysis. Academic Press, 1998, vol. 10.

[7] D. Donoho and I. Johnstone, "Ideal spatial adaptation via wavelet shrinkage," Biometrika, vol. 81, pp. 425-455, 1994.

[8] S. Meignen and D.-H. Pham, "Retrieval of the modes of multicomponent signals from downsampled short-time fourier transform,” IEEE Transactions on Signal Processing, vol. 66, no. 23, pp. 6204-6215, 2018.

[9] D.-H. Pham and S. Meignen, "A novel thresholding technique for the denoising of multicomponent signals," in 2018 IEEE International Conference on Acoustics, Speech and Signal Processing (ICASSP). IEEE, 2018, pp. 4004-4008.

[10] G. Thakur and H.-T. Wu, "Synchrosqueezing-based recovery of instantaneous frequency from nonuniform samples," SIAM Journal on Mathematical Analysis, vol. 43, no. 5, p. 2078-2095, Jan 2011. [Online]. Available: http://dx.doi.org/10.1137/100798818

[11] T. Oberlin, S. Meignen, and V. Perrier, "Second-order synchrosqueezing transform or invertible reassignment? Towards ideal time-frequency representations," IEEE Transactions on Signal Processing, vol. 63, no. 5, pp. 1335-1344, March 2015.

[12] S. Mann and S. Haykin, “The chirplet transform: Physical considerations,” IEEE Transactions on Signal Processing, vol. 43, no. 11, pp. 2745-2761, 1995.

[13] E. Candes and D. Donoho, "Ridglets: the key to high-dimensional intermittency," Phil. Trans. R. Soc. Lond, pp. $2495-2509$.

[14] L. Cohen, Time-frequency Analysis: Theory and Applications. Upper Saddle River, NJ, USA: Prentice-Hall, Inc., 1995.

[15] R. A. Carmona, W. L. Hwang, and B. Torrésani, "Multiridge detection and time-frequency reconstruction," IEEE Transactions on Signal Processing, vol. 47, no. 2, pp. 480-492, 1999.

[16] M. A. Colominas, S. Meignen, and D.-H. Pham, "Time-frequency filtering based on model fitting in the time-frequency plane,” IEEE Signal Processing Letters, vol. 26, no. 5, pp. 660-664, 2019.

[17] I. Daubechies, J. Lu, and H.-T. Wu, "Synchrosqueezed wavelet transforms: an empirical mode decomposition-like tool," Applied and Computational Harmonic Analysis, vol. 30, no. 2, pp. 243-261, 2011.

[18] S. Meignen, D.-H. Pham, and S. McLaughlin, "On demodulation, ridge detection, and synchrosqueezing for multicomponent signals," IEEE Transactions on Signal Processing, vol. 65, no. 8, pp. 2093-2103, 2017.

[19] R. Behera, S. Meignen, and T. Oberlin, "Theoretical analysis of the second-order synchrosqueezing transform," Applied and Computational Harmonic Analysis, vol. 45, no. 2, pp. 379-404, 2018.

[20] T. F. Quatieri, Discrete-time speech signal processing: principles and practice. Pearson Education India, 2006.

[21] L. Sachs, Applied Statistics: A Handbook of Techniques. New York: Springer-Verlag, 1984, vol. p.253. 